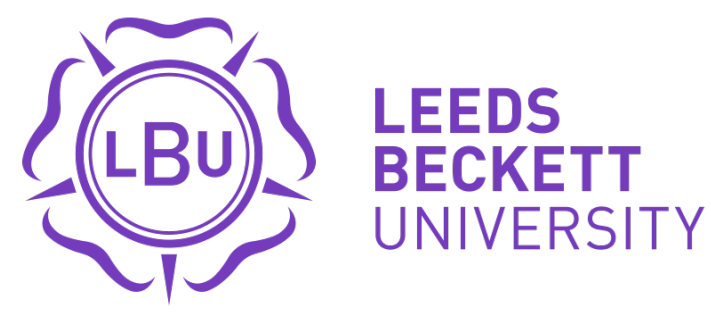

\title{
Citation:
}

Sanchez Sobrino, S and Monekosso, D and Remagnino, P and Vallejo, D and Glez-Morcillo, C (2019) Automatic recognition of physical exercises performed by stroke survivors to improve remote rehabilitation. In: The Second International Conference on Multimedia Analysis and Pattern Recognition, 09 May 2019 - 10 May 2019, Ho Chi Minh, Viet Nam. (In Press)

Link to Leeds Beckett Repository record:

https://eprints.leedsbeckett.ac.uk/id/eprint/5932/

Document Version:

Conference or Workshop Item (Accepted Version)

The aim of the Leeds Beckett Repository is to provide open access to our research, as required by funder policies and permitted by publishers and copyright law.

The Leeds Beckett repository holds a wide range of publications, each of which has been checked for copyright and the relevant embargo period has been applied by the Research Services team.

We operate on a standard take-down policy. If you are the author or publisher of an output and you would like it removed from the repository, please contact us and we will investigate on a case-by-case basis.

Each thesis in the repository has been cleared where necessary by the author for third party copyright. If you would like a thesis to be removed from the repository or believe there is an issue with copyright, please contact us on openaccess@leedsbeckett.ac.uk and we will investigate on a case-by-case basis. 


\section{Automatic recognition of physical exercises performed by stroke survivors to improve remote rehabilitation}

\author{
Santiago Schez-Sobrino \\ University of Castilla-La Mancha \\ Ciudad Real, Spain \\ Santiago.Sanchez@uclm.es
}

Dorothy N. Monekosso

Leeds Beckett University

Leeds, United Kingdom

D.N.Monekosso@leedsbeckett.ac.uk

\author{
Paolo Remagnino \\ Kingston University \\ Kingston upon Thames, United Kingdom \\ P.Remagnino@kingston.ac.uk
}

\author{
David Vallejo \\ University of Castilla-La Mancha \\ Ciudad Real, Spain \\ David.Vallejo@uclm.es
}

\author{
Carlos Glez-Morcillo \\ University of Castilla-La Mancha \\ Ciudad Real, Spain \\ Carlos.Gonzalez@uclm.es
}

\begin{abstract}
Strokes are the second cause of death and the third cause of disability in the world. Currently, there is not an actual cure for stroke victims, but physiotherapy can be used to restore as much mobility as possible until a plateau is reached again. However, performing these exercises implies that both patient and therapist are together in the same place, so that the latter can guide the former through a correct execution of physical exercises. This raises additional difficulties when it comes to continuously monitoring the recovery of stroke patients, due to the economic costs involved and the requirements of both geographical and temporal availability. These issues can be addressed by leveraging technology, specifically computer visionbased assistive systems and remote rehabilitation tools, so as to the affected person can check whether the exercises are being performed correctly. This paper is focused on the automatic classification of exercises, within the context of a gamificationbased remote rehabilitation tool used to automatically assess the performance of stroke patients when making physical rehabilitation. To this end, we use the DTW algorithm for analyzing and comparing open-ended motion curves, so that the exercises do not have to be fully performed until existing candidates can be matched. This increases the system flexibility and offers an interaction mechanism much more simple, which is usually a needed requirement by stroke patients.
\end{abstract}

Index Terms-Stroke, remote rehabilitation, automatic human activity recognition, dynamic time warping (DTW)

\section{INTRODUCTION}

According to the World Health Organization, strokes represent the world's second leading cause of death and the third of disability, respectively [1]. As an example, and only in the European continent, 165 million people are living with a brain disorder. In fact, it is estimated that one in three people suffer or will suffer from some type of neurological or psychiatric disorder [2]. Although there is no cure for this type of illness, physical rehabilitation is essential to improve the quality of life of those affected, as they have to deal with serious limitations when it comes to carry out activities of daily living, in which physical, cognitive, or social skills are involved. Unfortunately, the treatment of this type of disease has associated a high cost in terms of economic resources, since therapists are typically required to onsite monitor and supervise the performance of physical exercises carried out by the patients. For example, the overall cost to the European health system involves 800 billion euros per year, which represents a situation that is a burden on any health system. In addition, this problem is accentuated in low- and middle-income countries, where resource constraints are more pronounced.

This is why in recent years a relevant number of research works that focus on increasing the autonomy of the patient when performing therapies of rehabilitation [3] [4] has appeared, ensuring at all times adequate supervision by therapists. A typical scenario is one in which both the patient and the therapist make use of telemedicine tools, promoting the patient's recovery at home, reducing the costs of the rehabilitation process and offering a flexible solution which democratizes the access to physical rehabilitation.

From the patient's point of view, it is therefore essential to face the challenge of automatic human activity recognition. At research level, a possible solution involves the use of artificial systems based on computer vision [5] to obtain information about the physical movements that make up the rehabilitation routine. These systems use techniques such as skeleton tracking to obtain the position and rotation of the patient's bones at each instant of time, in order to assess whether the patient performs the rehabilitation exercises correctly. This evaluation can be undertaken through a comparison of the exercise performed by the patient and the same exercise performed by the therapist. This problem, which can be understood as a comparison of time series, can be dealt with by applying algorithms such as dynamic time warping (DTW) [6] or techniques such as automatic learning based on classifiers [7].

In addition to carrying out this automatic evaluation of exercises, artificial systems created to automate the process 
of physical rehabilitation of patients address the issue of motivation [8]. In fact, the ability to arouse interest in patients when using the artificial system is almost or more important than the effectiveness with which it evaluates and recognizes physical movements. In this sense, the use of gamification techniques, based on applying game dynamics in educational or professional environments to improve the results of using the tool, or serious games, which consists of creating games designed to achieve a goal other than purely playful, is becoming increasingly important in the context of remote rehabilitation tools [9].

The research work discussed in this article falls within the scope of a telemedicine tool designed for the remote rehabilitation of stroke patients. In particular, we will focus our work on the automatic classification of physical exercises using the open-end variant of the DTW algorithm [10]. The general objective is to increase the flexibility and accessibility of the system and enable patients to perform rehabilitation exercises without the need to indicate which specific exercise they will perform next. This is especially important for patients whose cognitive and functional abilities are so impaired that it is difficult to interact with the rehabilitation system itself. In these cases, the aim is to provide the system with an implicit interaction mechanism, so that it is capable of detecting when the patient starts and finishes an exercise, classifying it and automatically evaluating it to monitor the patient's evolution with respect to the assigned physical exercise routine.

The rest of the paper is structured as follows. Section II performs a review of the main research topics relevant to this work. Section III discusses the main features that the remote rehabilitation tool supports, i.e. the exercise performance assessment and the part on motivation. Section IV details how the classification of exercises is made. Finally, Section V summarizes the main contributions of this work and introduces possible lines of future work.

\section{RELATED WORK}

Patients who have stabilized after the first few days of a stroke should undergo rehabilitation exercises with a therapist so that they can regain most of their motor functions. In this context, rehabilitation exercises can be identified and classified automatically without the patients having to indicate the exercise they want to perform.

There are different algorithms that allow this classification. A variant of the DTW algorithm is presented in [11] to compare incomplete time series. The proposal, called openend DTW (OE-DTW), was successfully validated in this work through the classification of exercises used in the rehabilitation of stroke patients, depending on whether they were performed correctly or incorrectly. Also, in [12], the DTW algorithm is combined along with fuzzy logic to deal with the uncertainty of the data and make the comparison of the exercises performed by the patient with other reference exercises feasible. Moreover, there are also other tools that offer complete solutions when it comes to evaluating the execution of exercises and their classification, such as the one presented in [13], which proposes a system that divides the exercise recognition process into three stages where the initial and final posture of the patient is considered, as well as the comparison of the angular trajectories of the extremities involved in the exercise through the application of the DTW algorithm.

In addition, there are other approaches that propose the use of automatic learning to identify exercises performed by the patient, using techniques based on statistical models like Hidden Markov Models (HMM [14]), as proposed in [15], which presents a variant of this technique, named Hidden Semi-Markov Model (HSMM), to classify rehabilitation exercises and provide the patient with an assessment of their performance. In [16], a proposal is presented for automatic assessment of exercises with stroke patients, based on a first recognition stage through a Bayes classifier to reduce the number of candidates and then a second recognition using DTW or HMM; both were used with similar results. However, in [17], HMM is used instead of DTW to classify up to 13 warm up exercises, by averaging different HMMs into a single model. In [18], a technique based on Support Vector Machines (SVM [19]) and the algorithm of the k-nearest neighbors (kNN [20]) is used to recognize the possible gestures performed by the user using a Leap Motion ${ }^{\mathrm{TM}}$ device. In this line, in [21], prediction models based on decision trees are used to classify the joints of the human body using a Microsoft Kinect ${ }^{\mathrm{TM}}$ device from depth images captured by the sensor. Also, in [22], a novel technique is proposed using a fuzzy kernel motion classifier to solve the problem of overlapping between different parts of the body. Alternatively, in [23], a system was developed for the classification of exercises performed by patients with type 1 diabetes (T1D) according to their intensity, either aerobic or anaerobic. For this, the solution was based on the k-NN algorithm considering the heart rate, breath rate and peak acceleration during the movement of the patients.

The Table I shows a summary of the algorithms presented in the previous related works and what features did the authors implement using them. Therefore, the columns in the table would indicate whether the work included recognition of partially completed exercises and the type of information being classified, if any.

The alternatives discussed in this section offer a classification of exercises in different contexts. However, these classifications are undertaken once the exercises have been completed in full or attempts are made to classify them dividing the exercises into different stages. Thus, the proposal of this work intends to focus on offering an improvement to the remote rehabilitation of stroke survivors through the classification of exercises that have been partially performed, i.e., have not been completed.

\section{REMOTE REHABILITATION}

\section{A. Exercise Comparison}

The exercise analysis process is performed by comparing the exercise performed by the patient, with the same exercise saved as a reference and performed correctly by the 
TABLE I

COMPARISON TABLE CONSIDERING THE ALTERNATIVES PROPOSED IN THE RELATED WORKS AND THE FEATURES IMPLEMENTED BY THE AUTHORS

\begin{tabular}{|c|c|c|c|}
\hline Ref. & $\begin{array}{c}\text { Algorithm } \\
\text { used }\end{array}$ & $\begin{array}{c}\text { Partial } \\
\text { recognition }\end{array}$ & Classification \\
\hline$[11]$ & OE-DTW & Yes & Performance correctness \\
\hline$[12]$ & DTW, Fuzzy logic & No & Performance correctness \\
\hline$[13]$ & DTW & Yes & Exercise recognition \\
\hline$[15]$ & HSMM & No & Performance correctness \\
\hline$[16]$ & $\begin{array}{c}\text { Bayes classifier, } \\
\text { DTW/HMM }\end{array}$ & No & Performance correctness \\
\hline$[17]$ & Averaged HMMs & No & Exercise recognition \\
\hline$[18]$ & SVM, k-NN & No & Exercise recognition \\
\hline$[22]$ & $\begin{array}{c}\text { Fuzzy kernel } \\
\text { motion classifier }\end{array}$ & No & Exercise recognition \\
\hline$[23]$ & k-NN & Yes & Performance intensity \\
\hline
\end{tabular}

therapist. Specifically, the positions of the joints involved in the movement over time are compared. These positions are represented by a 3-tuple associated with a given point in the three-dimensional space, so that $(x, y, z) \in \mathbb{R}^{3}$, which are obtained through a camera with a depth sensor. In the specific case of this work, a Microsoft Kinect ${ }^{\mathrm{TM}}$ device has been used to help in the stage of recognition of the human skeleton.

The set of positions over time can be represented by a path or curve, thus simplifying the problem to a comparison of time series. To do this, the DTW algorithm allows this time series comparison, providing results independent of the time difference between the two sequences.

The DTW algorithm provides numerical results after comparison of both exercises such that dist $\in \mathbb{Q}_{>0}$. Internally, this value indicates the distance between the two curves, so the closer this value is to 0 , the greater the similarity between the two exercises, which means that there would be no significant differences between the two curves. This value is calculated by the algorithm using as a metric the Euclidean distance between the curves generated by both exercises, for the positions along the $\mathrm{X}, \mathrm{Y}$ and $\mathrm{Z}$ axes.

The value calculated as a result of applying the DTW algorithm is not restricted by an upper limit, so an added difficulty arises when interpreting these results. Therefore, it is necessary to perform a previous calibration step to define a set of intervals to help the interpretation of the results. This calibration is previously conducted by the therapist, executing some of the exercises of the patient's routine correctly and incorrectly (e.g. staying still), to obtain the minimum and maximum distances between curves. From these distances, three confidence intervals are calculated associated with the feedback messages provided to the user during the performance of the exercises. Thus, if the distances obtained by applying the DTW algorithm to the calibration exercise are $d_{r}$, exercise performed correctly, and $d_{w}$, exercise performed incorrectly, the confidence intervals would be calculated by obtaining a margin of error, $e$, that serves to relax the interpretation of the results obtained by the patient when performing the exercises. From this margin of error, the lower and upper limits of the intervals would then be calculated from the addition between the correct and incorrect distances divided by the number of intervals we want to define (which in the case of this work is 3), as shown in (1). Thus, the first interval would indicate a correct execution of the exercise, the second interval an acceptable execution and the third interval an incorrect execution. In this way, the feedback provided to the patients in those intervals is discretized, depending on how they performs the exercises.

$$
\begin{aligned}
d_{\text {sum }} & =d_{w}+d_{r} \\
d_{e} & =\frac{d_{\text {sum }}}{3} \\
{\left[0, d_{e}\right] \cup\left(d_{e}, d_{\text {sum }}\right.} & \left.-d_{e}\right) \cup\left[d_{\text {sum }}-d_{e}, \infty\right)
\end{aligned}
$$

On the other hand, the positions obtained from the joints must be normalized to avoid possible physical errors caused by a change of location of the patient or therapist with respect to the camera. For this purpose, these positions are recalculated with respect to one of the joints of the individual that does not affect the movement of the exercise, by default, the joint found at the base of the neck. This allows both calibration and reference exercises to be recorded remotely by the therapist for the patient to repeat, making it possible to compare the two performances.

\section{B. Motivation and Engagement}

Given the nature of a telemedicine tool where the patient can use it from home, it is essential to increase their motivation in order to prevent them from abandoning their exercises and periodically completing them. To this end, the tool incorporates different gamification mechanisms aimed at offering the patients a feeling of progression each time they perform their rehabilitation exercises. Fig. 1 shows a snapshot of the tool running as a web application with these available gamification mechanisms displayed in the main view.

Progression is shown in the tool through various visual indicators constantly available such as experience bars, levels, scores and achievements. In addition, after performing the exercises and analyzing the execution, the patients are given a star rating to help them understand how they performed the exercise, obtaining up to three stars if the exercise is performed correctly or one star if it is incorrect.

The experience bar is filled in from the scores obtained by the patients during their exercises. These points are accumulated until $100 \%$ of the experience bar is completed, which will reward the patient with a new level. The formula used to calculate the progression attends to the form $x_{i}=x_{\text {base }} \cdot t^{1.5}$, so that $x$ is the total amount of experience required to reach the $i$ level, $x_{\text {base }}$ is a minimum amount of experience, $t$ is the next level to reach and 1.5 is a constant used to increase the difficulty necessary to reach the next levels exponentially. The variable $x_{\text {base }}$ is only used to go from level 1 to level 2, since in the following levels this variable will be established to the total score accumulated by the patient in a single session, in order to adjust the difficulty for the following levels. 

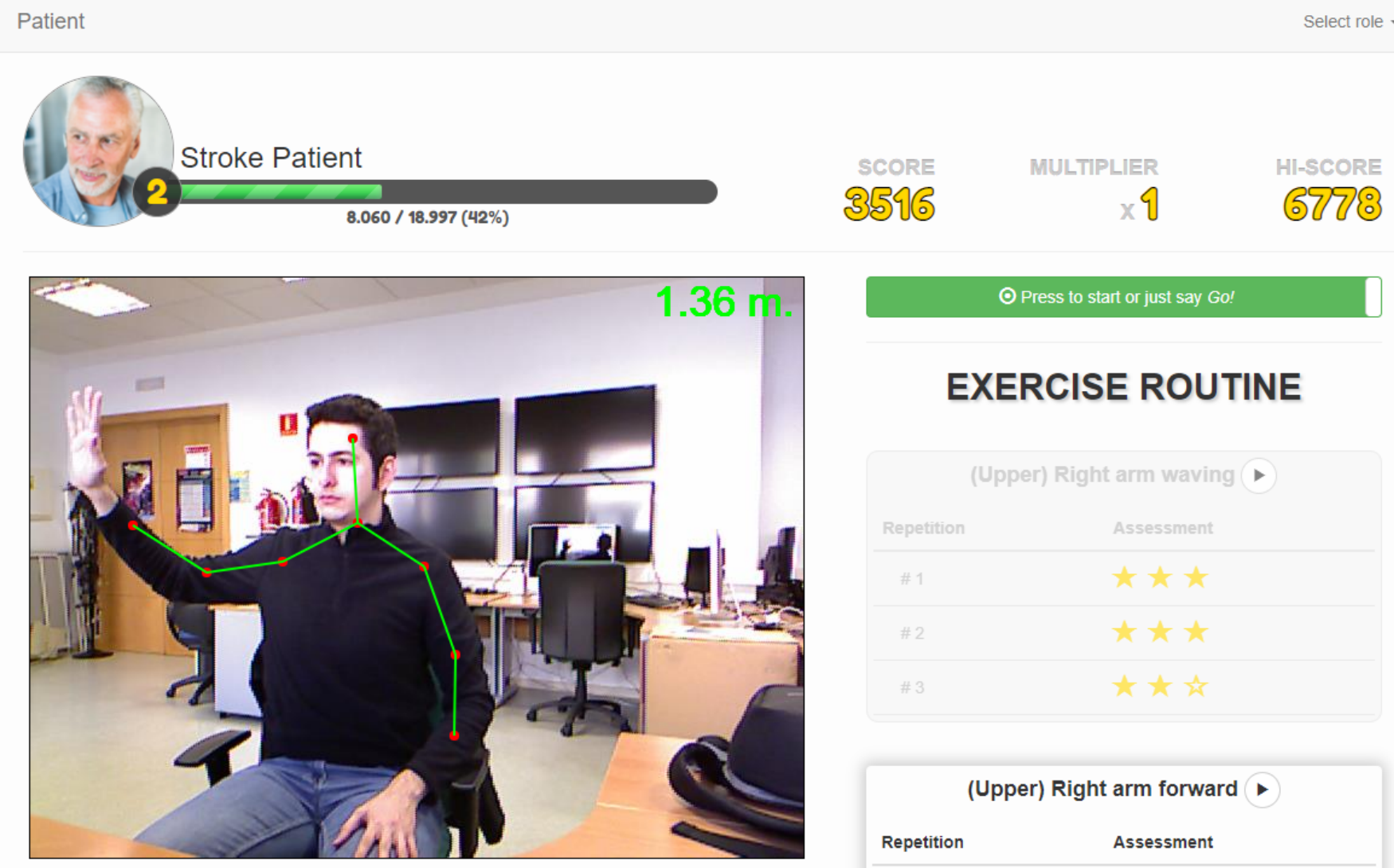

[10:38:36] Repetition performed. You obtained 0 points!
[10:36:23] Repetition performed. You obtained 888 points!
[10:36:12] Repetition performed. You obtained 1742 points!

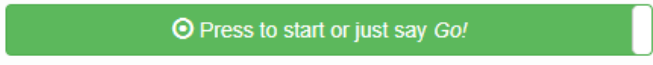

\section{EXERCISE ROUTINE}

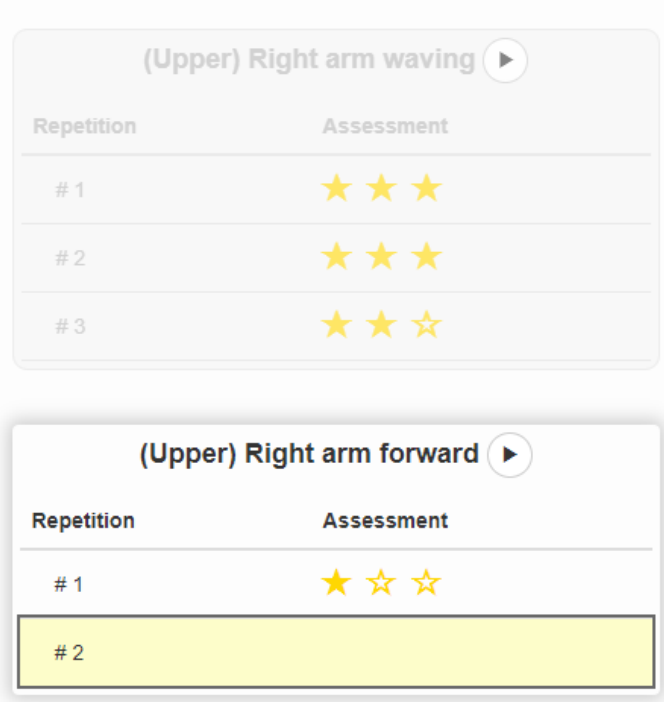

Fig. 1. Snapshot of the remote rehabilitation tool after a user had performed some repetitions for two assigned exercises.

The scores are calculated according to how the exercises are performed, obtaining all the points of the exercise if the system recognizes that the execution falls into the first of the confidence intervals, and decreasing as the result moves away from that interval. In addition, the patients can improve the scores obtained by applying multipliers if they achieve continuous streaks of exercises performed correctly.

The levels are the main objective to be reached by the patient, since they allow to unblock different achievements that demonstrate a real progression in his rehabilitation. The relationship between the achievements that are unlocked when reaching each level is defined by the therapist according to the evolution expected by the patient.

\section{EXERCISE RECOGNITION}

The telemedicine tool proposed in this work allows the comparison of the exercises performed by the patient with the same ones previously recorded by the therapist and used as reference (i.e. "gold standard") through the application of the DTW algorithm. To do this, the patient must select the exercise to be performed in the tool, interacting through voice commands or the user interface. This way of selecting the exercises to be performed adds certain difficulties to patients with reduced mobility who cannot interact with the system or who present severe cognitive problems that affect speech.

The DTW algorithm makes it possible to compare complete time series, thus assuming that the exercise that the patient has to perform has been completed completely before being compared with the reference exercise. The variant of this algorithm, called open-end DTW (OE-DTW) and originally published by Tormene et al. [11], makes it possible to compare incomplete time series, in addition to providing the percentage of coincidence between the two curves obtained so far. In the context of this work, the variant makes it possible to compare exercises whose execution has been started but which have not yet been fully completed. This makes it easier for the patients to perform the exercises without having to explicitly indicate which ones they wants to perform.

The tool integrates the implementation of the OE-DTW algorithm found in the statistical package available for $\mathrm{R}$ [10] [11]. During the use of the tool, the patient would start the movement of the exercise he/she wants to perform and the 
system would start to detect the exercise being performed. To do this, the system periodically compares the positions of the joints with those stored for the existing reference exercises. When an optimal candidate is found, that candidate exercise is marked as the definitive one and the patient is informed of the performance assessment for that exercise when completed.

For example, considering a sample setup where the patient has to perform repetitions for up to three different exercises (e.g. waving the right arm, lifting the right arm and moving the right arm forward), the implementation would have to find the best match while the patient perform the exercise over time. Therefore, when the user starts to perform a repetition for the exercise where he/she has to wave the right arm, an OE-DTW process is run to try to classify the movement from the $(x, y, z)$ positions of each joint, detected until that time instant. After this, several comparisons are made in parallel against each of the reference exercises defined above. The minimum distance obtained after applying the algorithm with the three reference exercises will be the one that indicates the exercise that has been recognized.

In Fig. 2 are shown the distances obtained over time after applying the OE-DTW algorithm against the three reference exercises. It can be observed how, during the first seconds of execution of the movement (i.e. time instants from the second 0 to 2), similar values are obtained because the three exercises start the movement in a similar way. From the second 4 , the values of the exercise "right arm forward" begin to distance themselves from the other two due to differences in execution. Finally, the exercise is correctly classified from second 9 onwards, with distance values lower than the other two exercises.

The algorithm is executed in parallel as many times as possible, obtaining a greater precision and speed in the classification as the hardware performance increases. The equipment where the tests were conducted, a MacBook Pro Mid-2015 (Intel Code i7@2.8 GHz), has allowed an average number of executions of 1.33 times per second. It should be noted that the same computer also performs other tasks related to the rehabilitation tool, such as communicating with the capture device to get information from the skeleton, process that information and send it to the web application for viewing.

\section{CONCLUSions}

We have presented a solution for the automatic recognition of physical exercises within the context of a gamificationbased remote rehabilitation system used by stroke survivors and therapists. Thanks to the use of the open-end variant of the DTW algorithm, this system is able to automatically classify, in real-time, the exercises that define the rehabilitation routines performed by patients. Thus, there is no need for a patient to particularly specify what exercise he/she is going to do next, which increases the system flexibility and accessibility if patients with severe difficulties to interact with this kind of tools are considered.

The conducted experimental results show the feasibility of using the OE-DTW algorithm for classifying exercises. To

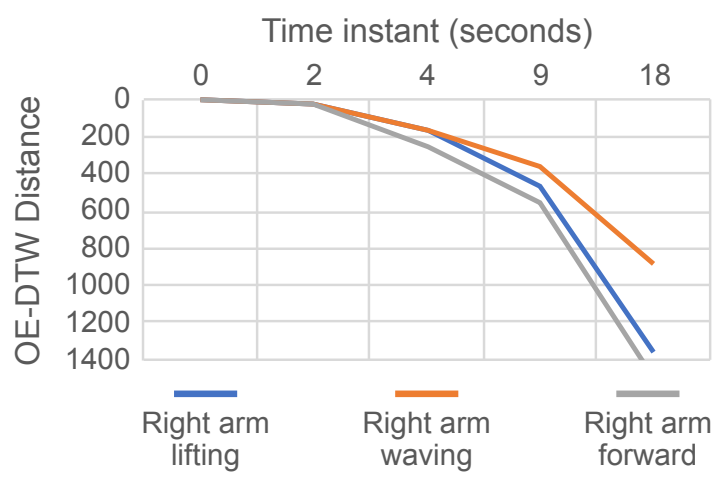

Fig. 2. Chart showing the obtained OE-DTW distances over time for the three reference exercises defined in the text.

this end, the implementation made in the rehabilitation tool has been introduced through an example where a user had to perform one of the exercises of the assigned routine, which contained a total of three different exercises. The results show how the exercise performed is successfully recognized after applying the algorithm in real time while the user performs the movement.

Our current research work is now focused on how to personalize physical rehabilitation for patients affected by neurological diseases. We aim to establish a methodology for precision rehabilitation through a co-creation and participatory approach between clinicians and patients, adopting an approach that promotes cost-efficient personalized rehabilitation in order to treat patients more effectively, in the context of neurological diseases. Pattern recognition will play an essential role when establishing customized rehabilitation routines depending on each patient condition and their ability to adjust rehabilitation treatments.

\section{ACKNOWLEDGEMENTS}

This research work has been partially funded by Instituto de Salud Carlos III through the project DTS18/00122 (cofunded by European Regional Development Fund/European Social Fund "Investing in your future") and Translate MedTech (Leeds City Region, UK).

\section{REFERENCES}

[1] W. Johnson, O. Onuma, and S. Sachdeva, "Stroke: a global response is needed," Bulletin of the World Health Organization, vol. 2016, no. 94, p. 634, 2016.

[2] V. L. Feigin, R. V. Krishnamurthi, P. Parmar, B. Norrving, G. A. Mensah, D. A. Bennett, S. Barker-Collo, A. E. Moran, R. L. Sacco, T. Truelsen et al., "Update on the global burden of ischemic and hemorrhagic stroke in 1990-2013: the gbd 2013 study," Neuroepidemiology, vol. 45, no. 3, pp. 161-176, 2015.

[3] S. Morales-Vidal and S. Ruland, "Telemedicine in stroke care and rehabilitation," Topics in stroke rehabilitation, vol. 20, no. 2, pp. 101107, 2013.

[4] N. van den Berg, M. Schumann, K. Kraft, and W. Hoffmann, "Telemedicine and telecare for older patients-a systematic review," Maturitas, vol. 73, no. 2, pp. 94-114, 2012.

[5] S.-R. Ke, H. Thuc, Y.-J. Lee, J.-N. Hwang, J.-H. Yoo, and K.-H. Choi, "A review on video-based human activity recognition," Computers, vol. 2, no. 2 , pp. 88-131, 2013. 
[6] S. Sempena, N. U. Maulidevi, and P. R. Aryan, "Human action recognition using dynamic time warping," in International Conference on Electrical Engineering and Informatics (ICEEI). IEEE, 2011, pp. 1-5.

[7] P. Turaga, R. Chellappa, V. S. Subrahmanian, and O. Udrea, "Machine recognition of human activities: A survey," IEEE Transactions on Circuits and Systems for Video technology, vol. 18, no. 11, p. 1473, 2008.

[8] G. Alankus, A. Lazar, M. May, and C. Kelleher, "Towards customizable games for stroke rehabilitation," in Proceedings of the SIGCHI Conference on Human Factors in Computing Systems. ACM, 2010, pp. 2113-2122.

[9] S. McCallum, "Gamification and serious games for personalized health," Stud Health Technol Inform, vol. 177, no. 2012, pp. 85-96, 2012.

[10] T. Giorgino et al., "Computing and visualizing dynamic time warping alignments in r: the dtw package," Journal of statistical Software, vol. 31, no. 7, pp. 1-24, 2009.

[11] P. Tormene, T. Giorgino, S. Quaglini, and M. Stefanelli, "Matching incomplete time series with dynamic time warping: an algorithm and an application to post-stroke rehabilitation," Artificial Intelligence in Medicine, vol. 45, no. 1, pp. 11-34, 2009.

[12] C. J. Su, C. Y. Chiang, and J. Y. Huang, "Kinect-enabled home-based rehabilitation system using Dynamic Time Warping and fuzzy logic," Applied Soft Computing, vol. 22, no. November 2014, pp. 652-666, 2014.

[13] D. Antón, A. Goñi, and A. Illarramendi, "Exercise Recognition for Kinect-based Telerehabilitation," Methods of Information in Medicine, vol. 54, no. 02, pp. 145-155, 2015.

[14] L. E. Baum and T. Petrie, "Statistical inference for probabilistic functions of finite state Markov chains," The annals of mathematical statistics, vol. 37, no. 6, pp. 1554-1563, 1966.

[15] M. Capecci, M. G. Ceravolo, F. Ferracuti, S. Iarlori, V. Kyrki, A. Monteriù, L. Romeo, and F. Verdini, "A Hidden Semi-Markov Model based approach for rehabilitation exercise assessment," Journal of Biomedical Informatics, vol. 78, no. February 2018, pp. 1-11, 2018.

[16] R. D. Willmann, G. Lanfermann, P. Saini, A. Timmermans, J. te Vrugt, and S. Winter, "Home Stroke Rehabilitation for the Upper Limbs," in 2007 29th Annual International Conference of the IEEE Engineering in Medicine and Biology Society. IEEE, 2007, pp. 4015-4018.

[17] A. Postawka, "Exercise Recognition Using Averaged Hidden Markov Models," in International Conference on Artificial Intelligence and Soft Computing (ICAISC 2017), 2017, pp. 137-147.

[18] W. J. Li, C. Y. Hsieh, L. F. Lin, and W. C. Chu, "Hand gesture recognition for post-stroke rehabilitation using leap motion," in Proceedings of the 3rd IEEE International Conference on Applied System Innovation (ICASI). IEEE, 2017, pp. 386-388.

[19] C. Cortes and V. Vapnik, "Support-vector networks," Machine learning, vol. 20, no. 3, pp. 273-297, 1995.

[20] N. S. Altman, "An Introduction to Kernel and Nearest-Neighbor Nonparametric Regression," The American Statistician, vol. 46, no. 3, pp. 175-185, 1992.

[21] J. Shotton, A. Fitzgibbon, M. Cook, T. Sharp, M. Finocchio, R. Moore, A. Kipman, and A. Blake, "Real-time human pose recognition in parts from single depth images," in Proceedings of the 21st IEEE Computer Vision and Pattern Recognition (CVPR), 2011, pp. 1297-1304.

[22] Z. Zhang, L. Liparulo, M. Panella, X. Gu, and Q. Fang, "A Fuzzy Kernel Motion Classifier for Autonomous Stroke Rehabilitation," IEEE Journal of Biomedical and Health Informatics, vol. 20, no. 3, pp. 893-901, 2016.

[23] K. Turksoy, T. M. L. Paulino, D. P. Zaharieva, L. Yavelberg, V. Jamnik, M. C. Riddell, and A. Cinar, "Classification of Physical Activity: Information to Artificial Pancreas Control Systems in Real Time," Journal of diabetes science and technology, vol. 9, no. 6, pp. 12001207, 2015. 